\title{
Intervention to improve social and family support for caregivers of dependent patients: ICIAS study protocol
}

Magdalena Rosell-Murphy ${ }^{*}$, Josep Ma Bonet-Simó ${ }^{2}$, Esther Baena ${ }^{2}$, Gemma Prieto $^{3}$, Eva Bellerino ${ }^{4}$, Francesc Solé ${ }^{5}$, Montserrat Rubio ${ }^{6}$, llona Krier ${ }^{4}$, Pascuala Torres ${ }^{6}$, Sonia Mimoso ${ }^{6}$ and the ICIAS research group

\begin{abstract}
Background: Despite the existence of formal professional support services, informal support (mainly family members) continues to be the main source of eldercare, especially for those who are dependent or disabled. Professionals on the primary health care are the ideal choice to educate, provide psychological support, and help to mobilize social resources available to the informal caregiver.

Controversy remains concerning the efficiency of multiple interventions, taking a holistic approach to both the patient and caregiver, and optimum utilization of the available community resources. For this reason our goal is to assess whether an intervention designed to improve the social support for caregivers effectively decreases caregivers burden and improves their quality of life.
\end{abstract}

Methods/design: Design: Controlled, multicentre, community intervention trial, with patients and their caregivers randomized to the intervention or control group according to their assigned Primary Health Care Team (PHCT). Study area: Primary Health Care network (9 PHCTs).

Study participants: Primary informal caregivers of patients receiving home health care from participating PHCTs. Sample: Required sample size is 282 caregivers (141 from PHCTs randomized to the intervention group and 141 from PHCTs randomized to the control group.

Intervention: a) PHCT professionals: standardized training to implement caregivers intervention. b) Caregivers: 1 individualized counselling session, 1 family session, and 4 educational group sessions conducted by participating PHCT professionals; in addition to usual home health care visits, periodic telephone follow-up contact and unlimited telephone support.

Control: Caregivers and dependent patients: usual home health care, consisting of bimonthly scheduled visits, follow-up as needed, and additional attention upon request.

Data analysis

Dependent variables: Caregiver burden (short-form Zarit test), caregivers' social support (Medical Outcomes Study), and caregivers' reported quality of life (SF-12)

Independent variables: a) Caregiver: sociodemographic data, Goldberg Scale, Apgar family questionnaire, Holmes and Rahe Psychosocial Stress Scale, number of chronic diseases. b) Dependent patient: sociodemographic data, level of dependency (Barthel Index), cognitive impairment (Pfeiffer test).

(Continued on next page)

\footnotetext{
*Correspondence: mrosell.mn.ics@gencat.cat

${ }^{1}$ Primary Care Team, Serraparera, Institut Català de la Salut. Cerdanyola del

Vallès, Barcelona 08290, Spain

Full list of author information is available at the end of the article
} 
(Continued from previous page)

Discussion: If the intervention intended to improve social and family support is effective in reducing the burden on primary informal caregivers of dependent patients, this model can be readily applied throughout usual PHCT clinical practice.

Trial registration: Clinical trials registrar: NCT02065427

Keywords: Caregiver burden, Social support, Primary health care

\section{Background}

Longer life expectancy not only increases the total number of elderly individuals but also raises the level of dependency in this age group [1]. Dependency leads to a physical decline that brings with it a need for psychosocial and health care services, coinciding with reduced income in retirement and a lack of adaptability to new situations that put the elderly person at a disadvantage in our rapidly changing world [2].

Despite the existence of formal professional support services, informal support continues to be the main source of eldercare, especially for those who are dependent or disabled [2,3]. Informal support includes the care and services provided by individuals, agencies, and networks other than formal services for the elderly who have some degree of psychophysical dependence; informal caregivers assist with basic and functional needs of daily living during most of the day, without economic compensation for this task [4].

Family members are the main source of informal support for the dependent person, and their support is a clear predictor of the patient's ability to remain in the home community and delay -or avoid- institutionalization. The person who takes the major responsibility for this care is defined as the primary caregiver for the dependent patient $[5,6]$.

A key concern for the primary caregiver is the excessive stress defined as caregiver burden [1-3], which has both objective and subjective components. The objective components are the demands, in the broadest sense of the term, to which the caregiver is exposed because of caring for the dependent person. The subjective components include the way the caregiver perceives the caregiving tasks, and specifically his or her emotional response to the experience of caring for a family member. When the emotional involvement is very intense, frequent, or longlasting, the caregiver's health and behaviour may be affected $[7,8]$. The evaluation of psychological well-being, a central component of health-related quality of life (HRQL), is also considered important [9]. Some studies have suggested that this indicator can be improved even when caregivers are excessively stressed, and that research on the health and well-being of caregivers must be complemented with assessments of quality of life or related aspects [10].

A study of informal caregivers in our primary care context [11] assessed the existence and causes of caregiver burden, along with the consequences for the patients, and observed that the overwhelmed caregiver has poor social support, cares for a severely dependent patient, and has been filling this role for a long time. In that study, $66.5 \%$ of caregiver burden was due to insufficient social support and variables related to the dependent patient, including age, cognitive status, and degree of dependency.

The adequacy of social support that the caregiver receives is related with the feeling of burden [12]. An inverse relationship has been reported between scores on the Medical Outcomes Study (MOS) test [13,14] which evaluates the social support received, and the Zarit test, which measures caregiver burden. Of all the factors related to caregiver burden, the easiest to modify is social support. Reinforcing social support reduces caregiver burden and improves quality of life. Therefore, an appropriate intervention in this dimension could help the caregiver and other family members, providing tools to confront the changes and respond more effectively to crises resulting from the deterioration of the dependent patient's status.

In order to sustain the activities of informal caregivers, it is important to attempt to limit or decrease their stress, strengthening their psychological well-being to improve their quality of life. Structured external interventions can improve the support available to informal caregivers, according to numerous recent studies focussed on caregivers for dependent patients in a home health care programme [8] with cerebrovascular accident [15,16] dementia [17-24], and schizophrenia [25].

Professionals on the primary health care team (PHCT) are the ideal choice to educate, provide psychological support, and help to mobilize social resources available to the informal caregiver. The PHCT doctor or nurse has a long-term relationship with the patient that also allows familiarity with the caregiver and care context and the opportunity to offer individualized intervention according to individual needs [26,27].

Various studies with different interventions designed to reduce or prevent caregiver exhaustion have been described [1]. Interventions focussed only on information, support groups, or management of behavioural disorders have not proven their effectiveness. Psychosocial interventions that address multiple dimensions (information about the disease, organization of care needs, practical advice, skill-building for care management, decision- 
making) are most indicated. Finally, the most successful models of intervention have involved long-term support for informal caregivers over a period of years. In this regard, other family members are among the caregiver's most accessible resources for ongoing support over time.

The REACH Project is a multi-intervention study that incorporated individual counselling, self-help groups, family therapy, caregiver training, and technological support; it was effective in improving caregiver health and delaying the institutionalization of the dependent patient [27]. The REACH conclusions establish that the intervention with the greatest possibility of success is structured but also personalized to meet the specific needs of the caregiver [27]. Even so, controversy remains concerning the efficiency of multiple interventions, taking a holistic approach to both the patient and caregiver, and optimum utilization of the available community resources [28].

For these reasons, we designed an intervention directed to the caregiver that takes both an individual and family approach to reduce caregiver burden and can be readily implemented by the patient's assigned $\mathrm{PHCT}$.

\section{Hypothesis}

A multi-factor intervention involving the primary caregiver of a dependent patient, with the goal of improving his or her social and family support and carried out by the professionals of the patient's regular PHCT, will decrease the burden and increase quality of life for the primary caregiver.

\section{Objective}

\section{Primary objectives}

1. To determine whether an intervention carried out by primary health care professionals, focussed on the caregiver, the family group, and a self-help group will improve the social support perceived by the caregiver.

2. To evaluate the effectiveness of the intervention in decreasing the caregiver's burden.

3. To determine whether the intervention improves the caregiver's perceived quality of life.

\section{Secondary objectives}

1. To identify population subgroups in which the intervention is most effective, according to caregivers' reported burden and quality of life.

2. To identify factors that influence the effectiveness of the intervention.

\section{Methods/design \\ Design}

Controlled, multicentre, community intervention trial, with random assignment by PHCT group.

\section{Setting}

Nine primary care centres in two regions of Catalonia (Vallès Occidental and Vallès Oriental), Spain.

\section{Type of participants}

Informal primary caregivers for patients in the home health care program, identified from electronic medical records (eCAP database).

\section{Inclusion criteria}

Adults (older than 18 years) identified in the eCAP database as the primary caregiver for a dependent patient, and who act as caregivers without remuneration.

Both the caregivers and the dependent patients have an active clinical record in a participating primary care centre.

\section{Exclusion criteria}

1. Caregivers for intermittent periods, independently of the length of care provided.

2. Caregivers who have provided less than one year of ongoing care.

3. Caregivers with any communication problem (psychiatric disorders, etc.) that makes them difficult to interview.

\section{Study sample}

Size

To avoid contamination of the intervention, randomization was done at the PHCT level. In a descriptive study of 500 primary caregivers throughout Catalonia, we obtained a standard deviation (SD) of 7.32 on the Zarit short test, or a difference of 4 points between tests at the beginning and end of the intervention. For a simple random design, accepting an alpha risk of 0.05 and a beta risk of 0.20 in a two-tailed test and 0.2 loss to follow-up, the sample must include 128 participants, 64 in the intervention group and 64 controls. Considering an intraclass correlation coefficient of 0.05 and an average of 25 informal primary caregivers of ATDOM patients per PHCT, the design effect is 2.2; therefore, a total of 282 caregivers are required for the study (141 per group). Sample size was calculated using Epidat 3.1 software.

\section{Measurement of main outcome variables \\ Dependent variables}

Social support The 20-item MOS test [13,14], which contains one question about the social network and 19 items assessing four dimensions of social support, measures the caregiver's subjective perceptions of the amount and types of social support received. Responses use a five-point Likert scale, with an overall range of 19 
to 95 points; below the cut-off of 57 , the respondent's social support is considered insufficient [14].

Caregiver burden The short-form Zarit test [29] assesses this variable, using the cut-off score of 17 to determine the existence of burden, based on a published pilot study [30].

Caregiver perception of quality of life The Short-Form 12 (SF-12) health questionnaire [31,32] elicits information in two areas, physical and mental health, and yields a profile of the respondent's health status. The 12 responses are scored on a Likert scale. To interpret the score, values are standardized to population norms, so that 50 (SD 10) is the average for the general population. Lower values are considered worse than the reference population [33].

Measurement of secondary variables and effect modifiers Independent variables

a) The Caregiver:

- Sociodemographic data: age, sex, marital status, employment status, educational level

- Time in caregiver role (in months)

- Score on Goldberg anxiety and depression scales [34,35].

- Family data: Family structure: World Health Organization classification, depending on household composition [36].

Phase of family life cycle (World Health Organization classification, modified by De la Revilla [37]): Phase 1, Formation (from marriage until the birth of the first child); Phase $2 A$, Expansion from first child's birth to 11 years of age; Phase 2B, Expansion from the first child's 11th birthday to the birth of the last child; Phase 3, End of the Expansion (from the birth of the last child until the first child leaves home); Phase 4, Contraction (from the first child's departure until the last child leaves home); Phase 5, End of Contraction (from the last child's departure until the first spouse's death; and Phase 6, Dissolution (from the death of the first spouse until the other spouse dies). Social network: Defined as the people who contribute support or assistance to an individual or family, and taken from the response to the first question on the MOS test, which specifically addresses this question. Family function: The perceived functionality of the family unit, assessed using the Apgar family questionnaire [38], which has 5 questions with
3 possible responses. Each answer scores between 0 and 2 points, for a range of 0 to 10 . A total score of 7 or more points indicates a functional family and a lower score suggests family dysfunction.

- Stressful life events: The Holmes and Rahe Social Readjustment Rating Scale $[39,40]$ has 43 items that measure stressful life events in "life-change units" over the previous 12 months. Respondents scoring more than 150 life-change units are considered to be at risk.

b) The dependent patient

Sociodemographic data: age, sex, marital status, educational level. Degree of dependence (Barthel Index) for activities of daily living [41] at the time of study inclusion.

Cognitive status: Pfeiffer Test, $[42,43]$ consisting of 10 true-or-false questions. Between 0 and 2 errors is considered normal intellectual function, 3 to 7 errors indicates potential deterioration, and 8 to 10 errors a severe intellectual deficit.

Reason for inclusion in home health care programme [44]: Eligible patients are classified into target groups according to different minimum common criteria, published by the Catalan government's Department of Health and Social Security in 1996:a) Chronic disease criterion: persons affected by chronic, neurologic, respiratory, rheumatologic or other processes; b)Terminal conditions criterion: persons in terminal stage of neoplasm, AIDS, or geriatric health conditions;

c) Cognitive deterioration criterion: persons with a permanent cognitive deficit; d)At-risk criterion: persons included in health promotion and disease prevention programmes because of social isolation, advanced age, physical or psychosocial dependency, architectural or other barriers. Visual analogue scale (0 to 100) for emotional status Subjective health assessment (0 to 100 points).

\section{Conduct of the study}

From electronic medical records, a list was obtained of the individuals identified as the primary caregiver for patients receiving home health care services. A simple random sample was selected. The corresponding doctor and/ or nurse contacted the potential participants by telephone to explain the study and ask if they would be interested in participating. Interested caregivers were visited at home by an independent interviewer, who provided an information sheet about the study and requested signature of informed consent to participate. To avoid any potential bias, the interviewer was simple-blinded to the participant's group assignment. An administrator compiled the baseline data for all participants into a database for analysis. 
Once the 282 study participants were identified, they were randomly assigned by PHCT to the intervention or control group. All PHCTs randomized to implement the intervention received the following standardized training:

1. Seminar on neurolinguistic approaches that facilitate communication between health professionals and caregivers, helping the health care team improve their skills related to active listening and recognition of unexpressed needs.

2. Workshop on approaches to family care, providing tools needed to understand and work with the family life cycle, social support structures, and genograms.

3. Working session on family interviews and understanding family relationships and their implications for caregiving, with specific sessions on strategies that foster collaboration and synergy between family members.

4. Training on working with groups, conducted by primary care professionals with appropriate training and experience.

5. Practical workshops designed to standardize the intervention strategies to be used by all participating PHCTs.

\section{Caregiver intervention}

The intervention was designed to activate and strengthen the social support for each caregiver. There are four dimensions to social support: instrumental support, positive social interaction, informational/confidential support, and emotional support. Previous intervention studies have observed that differentiated approaches are needed to achieve improvements in knowledge, attitude, and quality of life. This led us to design a multifactorial intervention $[45,46]$, as follows:

a) Individualized intervention (90 minutes): One counselling session with each caregiver, providing information about formal public and private resources that are available, according to the needs in each case, including relevant materials and emotional support (instrumental/emotional dimensions). In these sessions, the caregiver's main concerns and problems in properly carrying out his or her care tasks are identified and prioritized. The individuals to be invited to the family intervention session are also identified.

b) Family Intervention (1-2 group sessions, 90 minutes each): Sessions address the informational/ confidential, emotional, and positive social interaction dimensions.

c) Group educational sessions (up to four 60-minute sessions, depending on caregiver availability):
Informational/confidential, emotional and positive social interaction dimensions are addressed in three-part sessions: informational presentations on theory, time for sharing and discussion, and a relaxation exercise. Individuals learn from their own experiences and cognitive models, and make changes based on new information and the experiences of others.

\section{Content outline}

- Session 1:

- Introduction. Objectives of the intervention

- Health education concerning the patients' disease processes, with the groups organized around similar diseases if possible.

- Sharing and discussion, taking advantage of the content of participant comments to prepare following sessions, offer answers to questions that arise, and provide emotional support.

- Relaxation exercise

- Session 2:

- Introduction. Objectives of the intervention

- Health education concerning aspects of self-care for the caregiver (e.g., posture, nutrition, rest), information from the social worker about available resources, and response to questions raised in Session 1.

- Sharing and discussion, taking advantage of the content of participant comments to prepare following sessions, offer answers to questions that arise, and provide emotional support.

- Relaxation exercise

- Session 3:

- Introduction. Objectives of the intervention

- Health education about additional aspects of self-care, as well as the patient's hygiene, mobility and nutrition, and response to questions raised in Session 2.

- Sharing and discussion, taking advantage of the content of participant comments to prepare the final group session, offer answers to questions that arise, and provide emotional support.

- Relaxation exercise

- Session 4:

- Introduction. Objectives of the intervention

- Health education concerning medications and safety (e.g., avoiding falls and accidents in the home, orthopaedic resources)

- Sharing, discussion, and emotional support

- Relaxation exercise and closing 
In addition to monthly telephone follow-up, participants receive a direct telephone number to consult with a health professional for support as needed.

\section{Control group}

Caregivers in the control group received the usual followup provided through home health care by their PHCT, which includes scheduled visits to the home and additional specific attention as requested. This is the established means of maintaining a relationship between the doctor, nurse, and caregiver to facilitate patient follow-up and progress.

Following normal practice, needed resources were offered and all activities for caregivers that were being offered prior to the study period continued to be made available.

\section{Follow-up}

When the intervention had been completed, an independent interviewer contacted all caregivers in both groups by telephone to conduct a final survey.

\section{Analysis strategy}

An initial comparability analysis ensured homogeneity between the populations of the intervention and control groups. Descriptive analysis of all variables will include the Student $t$ and Mann-Whitney tests to compare means between two categories, ANOVA to compare three categories, chi-square to compare two categorical variables, and chisquare test for trends when one of the variables is ordered.

To determine which factors can be associated with the intervention, binary or ordinal unconditional logistic regression will be used to assess categorical variables, adjusting for potential confounding factors and clinical variables of possible relevance. The dependent patient's death or institutionalization is considered an end-point of the study. All analyses will be based on intention to treat. All two-tailed statistical tests will use a 95\% confidence level. The software packages SPSS for Windows version 15, STATA and HLM 6 will be used for all analyses.

The work plan and timeline is detailed in Figure 1.

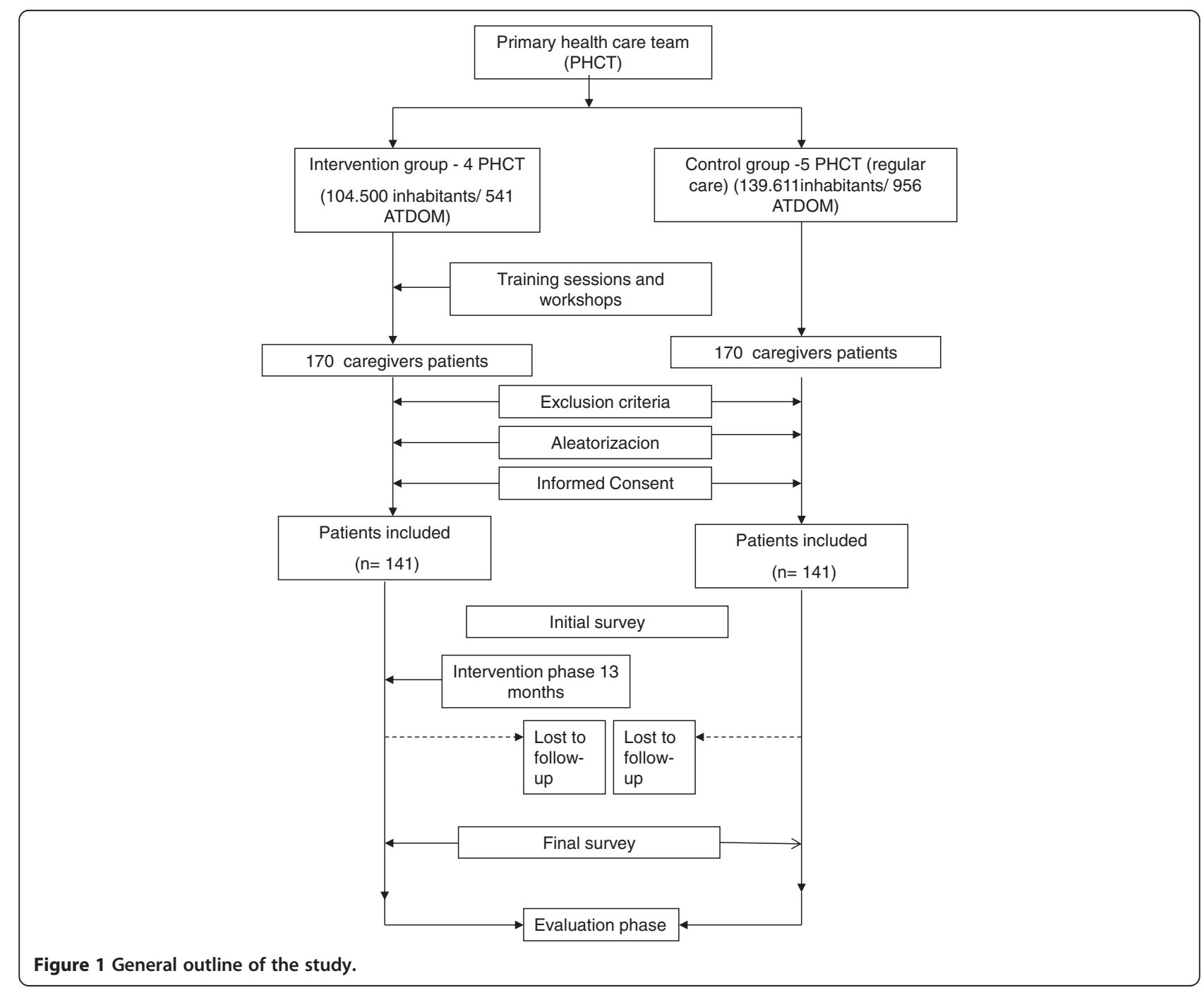




\section{Ethical aspects}

During the first direct contact with the caregiver, the project was presented together with a written request for voluntary participation. An information sheet provided the objectives and detailed characteristics of the study. A consent form described the general and specific ethical aspects related to the right to privacy, anonymity and confidentiality, to withdraw from the study without any penalty, and to receive study information. Participants were informed that, in order to guarantee the accuracy of study data, permission is required for researchers and health care authorities and/or members of the Ethics Committee for Clinical Research to gain access to their electronic medical records, with strict protection of the confidentiality of personal data. Only participants who provided signed informed consent were included.The protocol fully complies with Spain's data protection law (15/1999) and recent amendments (Royal decree 1720/2007) and was approved by the IDIAP Ethics Committee for Clinical Research (Comitè Etic d'Investigació Clinica del Institut de Investigació en Atenció Primària Jordi Gol) on March 2, 2009.

\section{Limitations}

Although 20\% loss to follow-up was calculated, the high mortality rates in the dependent population could exceed this estimate. The protocol provided for the addition of replacement caregivers if the initial sample decreased more than $20 \%$.

The caregiver assessments (interviews and tests) were blinded and independent for both groups, carried out by members of the research team not participating in the clinical care of the caregivers. There may be some variation between the participating PHCTs in the implementation of the intervention. Nonetheless, the training and workshops provided to PHCT teams in the intervention group are designed to standardize and contribute to homogeneity in implementing the intervention; usual care in the control group follows guidelines set out by the Catalan Health Service.

\section{Discussion}

The high levels of caregiver burden reported by other studies in our environment $[4,5]$ must sensitize us to the need to study the most appropriate instruments of caregiver support to diminish this burden. Family support decreases as years go by, and a negative first experience with caregiving can mean that the same person will not want to do it again and may prematurely choose the alternative of institutionalizing a dependent family member.

Numerous types of interventions aiming to improve patient care and caregivers self-care have been described, involving caregivers for patients with different chronic diseases and high dependency. Some studies demonstrate the efficacy of these interventions [12,13,15-24], although the results are moderate. All of this increases interest in establishing interventions that significantly improve the health of caregivers and consequently of the patients in their care.

This study applies various intervention strategies, in line with studies that demonstrate better response with combined interventions $[45,46]$. These include the individualized caregiver interview, directed at providing information, educating and modifying cognitive abilities to confront stressors and how they are perceived [45]; family interview to address the caregiver's environment; and group sessions for caregivers that focus on affective considerations and the emotional response of the caregiver [46]. Paying attention to the needs expressed by caregivers, together with the interventions proposed, facilitates the goal of improving family and social support.

If the final results obtained in our study show that increasing and strengthening the primary caregiver's social support improves quality of life and decreases caregiver burden, this will allow us to adopt a new perspective on home health care. A new strategy focussed on the family system and on caring both for the caregiver and dependent patient, providing more comprehensive support and better planning through the primary care system, may strengthen preventive efforts and improve care. The critical situations that occur when caring for dependent patients could also be addressed in ways that are more satisfactory for both the caregiver and patient, and even for the responsible health care professional. An intervention that focuses on procedural and behavioural changes supported by specific training for PHCT personnel will be relatively easy to extend throughout the primary care network.

\section{Competing interests}

The authors declare that they have no competing interests.

\section{Authors' contributions}

$M R$, JMB, and GP were responsible for the protocol design, project follow-up, and evaluation. FS, FN, EB, IK, AÁ, SK, MP, MR, EB, SM, and EV were responsible for developing materials for the caregivers. JMB, FS, EB, and IK were responsible for designing the intervention. EB was responsible for contact with the team leaders and coordinating the interviewers. FS, JMB, EB, and IK participated in the training of the key staff members of the PHCTs in the intervention group. PT, SK, MP, NA, DA, MRM, and SM were responsible for training related to group education sessions. All authors read and approved the final manuscript.

\section{Acknowledgements}

ICIAS RESEARCH GROUP: Montserrat Pons Segura, Silvia Kadar Laín, Ferran Naveros Almenara, Alicia Álvarez Lázaro, Adoración Alfonso Granero, Rosa M Martínez Luque, Eva Vinyallonga Flores, Núria Aznar. Without their interest and collaboration this study would not have been possible.

This project has been co-funded by the Regió Sanitària de Barcelona (fourth prize in research, 2009) and by CAMFIC (ninth prize in primary care research, 2009).

\section{Author details}

${ }^{1}$ Primary Care Team, Serraparera, Institut Català de la Salut. Cerdanyola del Vallès, Barcelona 08290, Spain. ${ }^{2}$ Primary Care Unit, Cerdanyola-Ripollet, Institut 
Català de la Salut. Cerdanyola del Vallès, Barcelona, Spain. ${ }^{3}$ Primary Care Agency, Avila, Spain. ${ }^{4}$ Primary Care Team, Ripollet, Institut Català de la Salut, Barcelona, Spain. ${ }^{5}$ Primary Care Team, Badia del Vallès, Institut Català de la Salut, Barcelona, Spain. ${ }^{6}$ Primary Care Team, Canaletes, Institut Català de la Salut. Cerdanyola del Vallès, Barcelona, Spain.

Received: 26 February 2014 Accepted: 12 March 2014

Published: 25 March 2014

\section{References}

1. Martin-Carrasco M, Martin MF, Valero CP, Millan PR, Garcia Cl, Montalbán SR, Vázquez AL, Piris SP, Vilanova MB: Effects of a psychological intervention in a primary health care center for caregivers dependent relatives: a randomized trial. Int J Geriatr Psychiatry 2009, 24:489-499.

2. Gutierrez Eizaguirre B: Formación de familias cuidadoras de personas mayores dependientes. Cuadernos de trabajo social 1998, 11:202-220. Universidad Complutense. Madrid.

3. Losada A, Pérez-Peñaranda A, Rodriguez-Sanchez E, Gomez-Marcos MA, Ballesteros-Rios C, Ramos-Carrera IR, La Torre MA C-d, Garcia-Ortiz L: Leisure and distress in caregivers for elderly patients. Arch Gerontol Geriatr 2010, 50(3):347-350

4. Badia Llach X, Lara Suriñach N, Roset Gamisans M: Calidad de vida, tiempo de dedicación y carga percibida por el cuidador principal informal del enfermo de Alzheimer. Aten Primaria 2004, 34:170-177.

5. Alonso Babarro A, Garrido Barral A, Díaz Ponce A, Casquero Ruiz R, Riera Pastor M: Perfil y sobrecarga de los cuidadores de pacientes con demencia incluidos en el programa ALOIS. Aten Primaria 2004, 33:61-66.

6. Woodford J, Farrand P, Richards D, Llewellyn D: Psychological treatments for common mental health problems experienced by informal carers of adults with chronic physical health conditions (Protocol). Systematic Reviews 2013, 2:9.

7. Argimon JM, Limón E, Abós T: Sobrecarga y calidad de vida de los cuidadores informales de pacientes discapacitados. Aten Primaria 2003, 32:84-85.

8. Robison J, Fortinsky R, Kleppinger A, Shugrue N, Porter M: A broader view of family caregiving: effects of caregiving and caregiver conditions on depressive symptoms, health, work, and social isolation. Gerontol $B$ Psychol Sci Soc Sci 2009, 64(6):788-798.

9. Coen RF, O' Boyle CA, Coakley D, Lawlor BA: Individual quality of life factors distinguishing low-burden and high-burden caregivers of dementia patients. Dement Geriatr Cogn Disord 2002, 13:164-170.

10. Chappell NL, Reid C: Burden and well-being among caregivers: examining the distinction. Gerontologist 2002, 42:772-780.

11. Ko Bae M, Bonet Simó JM, Rubio Villar M, Pons Segura M, Clotet Romero L, Ruiz Cámara M: Apoyo Social ¿mejora el cansancio del cuidador? Aten Primaria 2004, 34(extraordin):198.

12. Guerrero Caballero L, Ramos Blanes R, Alcolado Aranda A, López Dolcet MJ, Pons La Laguna L, Quesada Sabaté M: Programa de intervención multidisciplinaria para cuidadores de pacientes en atención domiciliaria. Gac Sanit 2008, 22(5):457-460.

13. Sherbourne CD, Stewart AL: The MOS social support survey. Soc Sci Med 1991, 32:705-714

14. Revilla Ahumada L, De La Luna Del Castillo J, Bailón Muñoz E, Medina Moruno I: Validación del cuestionario MOS de apoyo social en Atención Primaria. Medicina de Familia 2005, 6:10-14.

15. Larson J, Franzén-Dahlin A, Billing E, Arbin M, Murray V, Wredling R: The impact of the nurse led support and education programme for spouses of stroke patients: a randomized controlled trial. J Clin Nurs 2005, 14(8):95-1003.

16. Schure LM, Van den Heuvel ET, Stewart RE, Sanderman R, De Witte LP, Meyboom- de Jong B: Beyond stroke: description and evaluation of an effective intervention to support family caregivers of stroke patients. Patient Educ Couns 2006, 62(1):46-55.

17. Hébert R, Lévesque L, Vézina J, Lavoie JP, Ducharme F, Gendron C, Préville M, Voyer L, Dubois MF: Efficacy of a psychoeducative group program for caregivers of demented persons living at home: a randomized controlled trial. J Gerontol B Psychol Sci Soc Sci 2003, 58(1):58-67.

18. Glass TA, Berkman LF, Hiltunen EF, Furie K, Glymour MM, Fay ME, Ware J: The families in recovery from stroke trial (FIRST): primary study results. Psychosom Med 2004, 66:889-897.
19. Thompson C, Spilsbury K: WITHDRAWN: support for carers of people with Alzheimer's type dementia. Cochrane Database Syst Rev 2007, 3, CD000454.

20. Thompson C, Spilsbury K, Hall J, Birks Y, Barnes C, Adamson J: Systematic review of information and support interventions for caregivers of people with dementia. BMC Geriatr 2007, 7:18.

21. Brodaty H, Green A, Koschera A: Meta-analysis of psychosocial interventions for caregivers of people with dementia. J Am Geriatr Soc 2003, 51(5):657-664

22. Mittelman MS, Roth DL, Coon DW, Haley WE: Sustained benefit of supportive intervention for depressive symptoms in caregivers of patients with Alzheimer's disease. Am J Psychiatry 2004, 161:850-856.

23. Logiudice D, Waltrowicz W, Brown K, Burrows C, Ames D, Flicker L: Do memory clinics improve the quality of life of carers? A randomized pilot trial. Int J Geriatr Psychiatry 1999, 14:626-632.

24. Vinyoles E, Coppetti C, Cabezas C, Megido MJ, Espinàs J, Vila J, Montella N, Martínez A, Argimón JM, Grupo de Estudio CUIDA'L: Cuida'I: un ensayo clínico que evaluará la eficacia de una intervención en cuidadores de pacientes demenciados. Aten Primaria 2001, 27:49-53.

25. Magliano L, Fiorillo A, Malangone C, De Rosa C, Maj M: Patient functioning and family burden in a controlled, real-world trial of family psychoeducation for schizophrenia. Psychiatr Serv 2006, 57(12):1784-1791.

26. Beinart N, Weinman J, Wade D, Brady R: Caregiver burden and psychoeducational interventions in Alzheimer's disease: a review. Dement Geriatr Cogn Disord Extra 2012, 2:638-648.

27. Schulz R, Burgio L, Burns R, Eisdorfer C, Gallagher-Thompson D, Gitlin LN, Mahoney DF: Resources for enhancing Alzheimer's caregiver health (REACH): overview, site-specific outcomes, and future directions. Gerontologist 2003, 43:514-520.

28. Chiatti C, Masera F, Rimland JM, Cherubini A, Scarpino O, Spazzafumo L, Lattanzio F, UP-TECH research group: The UP-TECH project, an intervention to support caregivers of Alzheimer's disease patients in Italy: study protocol for a randomized controlled trial. Trials 2013, 14:155.

29. Regueiro Martínez AA, Pérez-Vázquez A, Gómara Villabona SM, Ferreiro Cruz MC: Escala de Zarit reducida para la sobrecarga del cuidador en atención primaria. Aten Primaria 2007, 39:185-188.

30. Breinbauer H, Vásquez H, Mayanz S, Guerra C, Millán T: Validación en Chile de la Escala de Sobrecarga del Cuidador de Zarit en sus versiones original y abreviada. Rev Méd Chile 2009, 137:657-665.

31. Gandek B, Ware JE, Aaronson NK, Apolone G, Bjorner JB, Brazier JE, Bullinger M, Kaasa S, Leplege A, Prieto L, Sullivan M: Cross-validation of item selection and scoring for the SF-12 Health Survey in nine countries: results from the IQOLA Project. International Quality of Life Assessment. J Clin Epidemiol 1998, 51(11):1171-1178.

32. Jenkinson C, Layte R, Jenkinson D, Lawrence K, Petersen S, Paice C, Stradling J: A shorter form health survey: can the SF-12 replicate results from the SF-36 in longitudinal studies? J Pub Health Med 1997, 19:179-186.

33. Schmidt S, Vilagut G, Garin O, Cunillera O, Tresserras R, Bruqulat P, Mompart A, Medina A, Ferrer M, Alonso J: Normas de referencia para el Cuestionario de Salud SF-12 versión 2 basadas en poblacion general de Cataluña. Med Clin (Barc) 2012, 139(14):613-625.

34. Goldberg D, Bridges K, Duncan-Jones P, Grayson D: Detecting anxiety and depression in general medical settings. BMJ 1988, 297:897-899.

35. Montón C, Pérez Echeverria MJ, Campos R, Garcia Campayo J, Lobo A: Escalas de ansiedad y depresión de Goldberg: una guía de entrevista eficaz para la detección del malestar psíquico. Aten Primaria 1993, 12(6):345-349.

36. De la Revilla L, Moreno A, Carrasco M, Fleitas L, Puertas F, Bailon: Bailón: Medicina familiar y práctica familiar. Libro de Ponencias del III Congreso de la Sociedad Andaluza de Medicina Familiar y Comunitaria. Granada; 1989.

37. De la Revilla L, Fleitas L: Instrumentos para el abordaje familiar. Atención longitudinal: el ciclo vital familiar. In Atención Primaria: conceptos, organización y práctica clínica. 4a. Edited by Martín Zurro A, Cano Pérez FJ. Spain: Elsevier (Barcelona); 1999:115-129.

38. Bellon JA: Cuestionario de función Familiar Apgar-Familiar. Aten Primaria 1996, 18:289-296.

39. Holmes TH, Rahe RH: The Social Readjustment Rating Scale. J Psychosom Res 1967, 11(2):213-218.

40. Rahe RH, Arthur RJ: Life change and illness studies: past history and future directions. J Human Stress 1978, 4(1):3-15.

41. Cid-Ruzafa J, Damián-Moreno JD: Valoración de la discapacidad física: el Indice de Barthel. Rev Esp Salud Publica 1997, 71:127-137. 
42. Pfeiffer E: A short portable mental status questionnaire for the assessment of organic brain deficit in elderly patients. J Am Geriatr Soc 1975, 23:433-441.

43. Martínez J, Dueñas R, Onís MC, Aguado C, Albert C, Luque R: Adaptación y validación al castellano del cuestionario de Pfeiffer (SPMSQ) para detectar la existencia de deterioro cognitivo en personas mayores de 65 años. Med Clin 2001, 117:129-134.

44. Generalitat de Catalunya, Servei Catala de la Salut: Programa de millora de l'atenció al domicili des de l'atenció primària de salut. Pla de Salut. Quadern núm.8. Departament de Sanitat and Seguretat Social; 1996.

45. Etters L, Goodall D, Harrison BE: Caregiver burden among dementia patient caregivers:A review of the literature. J Am Acad Nurse Pract 2008, 20(8):423-428.

46. Pinquart $M$, Sörensen $S$, Duberstein $P$ : How effective are interventions with caregivers? An updated meta-analysis. Gerontologist 2002, 42(3):356-372.

doi:10.1186/1471-2296-15-53

Cite this article as: Rosell-Murphy et al: Intervention to improve social and family support for caregivers of dependent patients: ICIAS study protocol. BMC Family Practice 2014 15:53.

\section{Submit your next manuscript to BioMed Central and take full advantage of:}

- Convenient online submission

- Thorough peer review

- No space constraints or color figure charges

- Immediate publication on acceptance

- Inclusion in PubMed, CAS, Scopus and Google Scholar

- Research which is freely available for redistribution 